\title{
Modelo de Gestión del Conocimiento en la prestación de asesoría financiera y tributaria a personas naturales en la parroquia Manta del cantón Manta
}

\section{Knowledge Management Model in the provision of financial and tax advice to citizens in the Manta Parish of Manta Canton}

\author{
Ing. Luis Miguel Lascano Intriago ${ }^{1}$ \\ lumilain@gmail.com \\ https://orcid.org/oooo-0002-9396-8939 \\ Eco. Olmedo Farfán, Msc. ${ }^{2}$ \\ olmedo.farfan@uteg.edu.ec \\ https://orcid.org/0000-0003-1385-6994
}

Recibido: 01/07/2020; Aceptado: 01/09/2020

\begin{abstract}
RESUMEN
Este trabajo de investigación ha sido realizado con el objetivo de establecer una relación de causalidad entre variables como la asesoría tributaria-financiera y el aumento de la recaudación tributaria y generación de plazas de empleo. Para el efecto se desarrolló una metodología econométrica que explique esta causalidad y se compruebe la hipótesis planteada acerca de los efectos positivos de brindar este tipo de servicios. Igualmente, se realizó una causalidad inversa, es decir, invirtiendo las variables. Se aplicó este modelo a la Corporación Financiera Nacional, sucursal Manta. De igual manera, también se analiza el efecto que pueda tener el contar con un mayor número de trabajadores en esta entidad bancaria pública en el volumen y montos de créditos que se ofrezcan. Los resultados finales mostraron que un mayor monto de crédito aprobado no necesariamente generaría un mayor número de plazas de empleo, pero sí un aumento de ingresos tributarios al Fisco.
\end{abstract}

Palabras clave: gestión, asesoría financiera, tributos, cantón Manta

\section{ABSTRACT}

The objective of the present research paper is to establish a causal relationship between variables such as financial-tax advisory services and an increase in tax collections as well with an increase in the level of employment. An econometric method is used to explain such causality and to prove the hypothesis: tax - financial services do have a positive relationship. Also, the variables are inverted in the model, thereby, an inverse causality approach is applied. The model described has been applied to the National Financial Corporation, Manta branch. Likewise, it also analyzes if having a higher number of employees at that branch would have an effect

\footnotetext{
${ }^{1}$ Universidad Tecnológica Empresarial de Guayaquil, Guayaquil, Ecuador.

2 Universidad Tecnológica Empresarial de Guayaquil, Guayaquil, Ecuador
} 


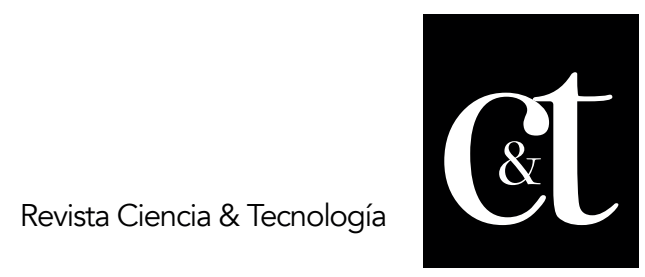

No. 28, 31 de octubre de 2020

ISSN impreso: 1390 - 6321

ISSN online: 2661 - 6734

in the volume of credit granted. Final results show no correlation between volume of credit approved and level of employment, but it does show a significant relationship with an increase in tax collections.

Keywords: management, financial advisory, taxes, Manta Canton

\section{Introducción}

El mercado financiero del Ecuador es cada vez más competitivo y se desarrolla en un ambiente cambiante. El usuario es más exigente con tiempos de respuesta y calidad del servicio que recibe por parte de las instituciones bancarias, aspecto que está legislado por el Código Orgánico Monetario y Financiero (Asamblea Nacional del Ecuador, 2014).

Estas exigencias, según Trujillo (2002) y Samaniego, Trujillo \& Martin (2006), conducen a que el sistema financiero enfrente nuevos retos y la oferta de un amplio portafolio de productos y servicios que los diferencie de otras entidades, siendo el diferenciador la calidad de servicio, lo cual a su vez es corroborado por Emery, Finnerty \& Stowe (2000).

Sin embargo, a pesar que las instituciones financieras mantienen plenamente diseñados sus productos y servicios crediticios para sus clientes (SRI, 2016), estos deben cumplir con las exigencias del órgano de control, respecto a la documentación que cada expediente de crédito debe mantener y del proceso de análisis crediticio que sigue, por lo que es preponderante que los prominentes solicitantes de créditos cumplan a cabalidad con los requisitos de cada operación crediticia solicitada. La información que puedan recibir contribuiría a reducir drásticamente la imposibilidad del impago o no retorno de los recursos entregados (Navarro, 2003; Estrada, 2012).

Sin embargo, más allá de las disposiciones establecidas por las instituciones financieras y los órganos de control, existen a su vez algunos determinantes que inciden en los procesos de crédito desde el punto de vista del prestamista que tienen que ver especialmente con la cultura financiera y crediticia de las personas naturales, y que contribuyen a aumentar el riesgo, como son el poco conocimiento de las ventajas del crédito y del uso apropiado de las herramientas de crédito, reticencia o desconfianza a recibir asesoramiento, percepciones sobre el sistema financiero, entre otros aspectos (Ismail, 2013).

Esta situación, común sobre todo en países no desarrollados, no es ajena al Ecuador, donde estudios realizados han demostrado la poca cultura financiera de la ciudadanía en general (Peñarreta, García \& Armas, 2019).

Este trabajo tiene como finalidad analizar la importancia que tiene, dentro del proceso de otorgamiento de créditos, la correcta presentación de la información a presentar por parte del cliente y del impacto favorable que tendría un proceso eficiente y eficaz de servicio de asesoría financiera-tributaria a las personas naturales en la parroquia Manta del Cantón Manta, siendo materia de estudio la información estadística de la Sucursal en Manta, de las Corporación Financiera Nacional (CFN). El objetivo es fomentar ese asesoramiento financiero-tributario a las personas naturales, potenciales clientes de la institución financiera bajo estudio, para así cuantificar, medir y mejorar los resultados financieros. 


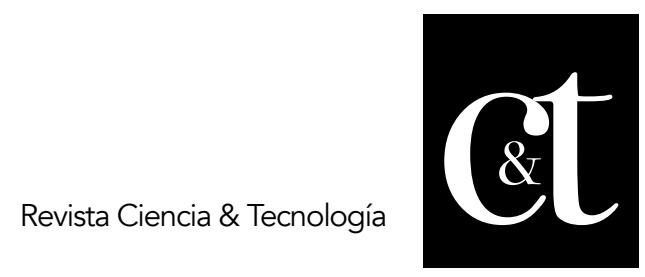

No. 28,31 de octubre de 2020

ISSN impreso: 1390 - 6321

ISSN online: 2661 - 6734

Cabe entonces, ejecutar un modelo de gestión del conocimiento en la prestación de servicios de asesoría, aplicando los principios y herramientas contables, financieras y fiscales, de manera que los potenciales clientes puedan auto realizarse un análisis profundo de su situación económica actual y futura, es decir de su capacidad de pago.

En efecto, el modelo de gestión del conocimiento de la prestación de asesoría financiera y tributaria a personas naturales en la parroquia Manta del cantón Manta, requiere de un análisis de regresión lineal simple con la finalidad de determinar una tendencia y correlación entre sus variables (Berenson, Levine \& Krehbiel, 2006).

\section{Metodología}

Por lo descrito y las características de este análisis, esta investigación es de carácter aplicado, de alcance descriptivo y de enfoque cuantitativo, puesto que se utilizaron las cifras disponibles de la Corporación Financiera Nacional que son de dominio público.

Con el uso de técnicas econométricas se estimaron modelos de regresión lineal simple para establecer relaciones funcionales de causalidad entre las variables objeto de estudio que son los montos de créditos aprobados por la CFN, como variable independiente y el número de operaciones crediticias, el nivel de empleo y el monto de impuestos generados a partir de los créditos, como variables dependientes. Se utilizó el paquete estadístico de Excel, con el que se estimaron también las pruebas diagnósticas necesarias para validar los modelos y los coeficientes de correlación entre las variables.

Las variables se analizaron de acuerdo a la investigación no experimental y los diseños transeccionales correlacionales. Las técnicas para la recolección de información que se utilizaron fueron: técnicas estadísticas; técnicas de investigación de campo y técnicas de análisis documental (Kon \& Storey, 2003).

Al finalizar el presente análisis, con la aplicación de las ecuaciones resultantes de los modelos econométricos mediante la regresión lineal, se pudo pronosticar lo siguiente: a) incremento de las operaciones de crédito directo para personas naturales; b) incremento de nuevas plazas de trabajo; y, c) estimación del aporte en tributos fiscales del segmento "personas naturales".

\section{Resultados}

Tomando en consideración que las variables dependientes son los números de trabajos que generan estos créditos productivos y el número de operaciones que en cada año se aprobaron y la independiente son los montos de los créditos aprobados, se pueden establecer unos modelos de regresión lineal simple, los cuales se plantean a continuación para cada par de variable dependiente vs la variable independiente.

\section{Regresión simple del número de operaciones vs montos de operaciones aprobadas}

Al analizar los datos de los montos y número de operaciones aprobadas para determinar si existe una correlación entre ambas variables, se obtiene: 
Tabla 1. Datos de los montos y números de operaciones aprobadas

\begin{tabular}{|c|c|c|}
\hline Periodo & $\begin{array}{c}\text { Operaciones (y) } \\
\text { (unidades) }\end{array}$ & $\begin{array}{c}\text { Montos (x) } \\
\text { (cientos de miles US\$) }\end{array}$ \\
\hline Año 2012 & 18 & 23,7 \\
\hline Año 2013 & 23 & 47,4 \\
\hline Año 2014 & 16 & 15,2 \\
\hline Año 2015 & 16 & 65,3 \\
\hline Año 2016 & 28 & 78,8 \\
\hline $\begin{array}{l}\text { Intercepto } \\
\text { Pendiente }\end{array}$ & $\begin{array}{l}14,51234 \\
0,12346\end{array}$ \\
\hline
\end{tabular}

Fuente: Corporación Financiera Nacional Banca Pública

Elaborado: Los autores

El pronóstico viene de la aplicación de ecuación (tendencia lineal) $Y t=a+b x$, donde (a) es igual el intercepto y (b) es la pendiente y la $(x)$ es igual al valor que se quiere determinar cómo pronósticos, en este caso por ejemplo, si se considera que el monto de operaciones de crédito a aprobar equivale a 90 (en cientos de miles de USD) que corresponde a 9 millones de US dólares, aplicando la ecuación de regresión, el número de operaciones ascendería a 26 en correlación a lo que se ha aprobado en el pasado.

El Gráfico 1 de la dispersión del número de operaciones vs montos de créditos aprobados muestra que estos estudios se basan en la creencia de que es posible identificar y/o cuantificar alguna relación funcional entre dos o más variables, en la gráfica de histograma se refleja una línea con pendiente positiva, lo cual quiere decir que su tendencia es creciente en el tiempo con un coeficiente de determinación $\mathrm{R}^{2}$ de 0,40479 lo que significa que los montos aprobados explican la variación observada en los números de operaciones en un $40,5 \%$.

\section{Dispersión Operaciones (y) vs Montos}

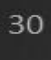

25

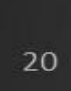

20

15

10

10

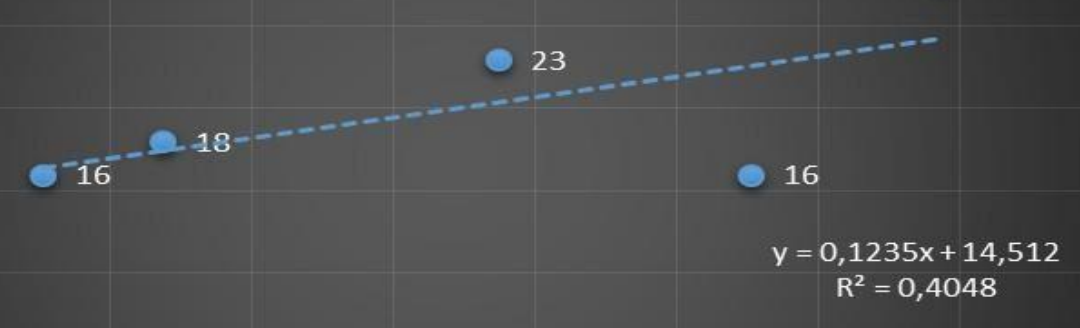

5

0

Gráfico 1. Dispersión número de operaciones vs montos de créditos aprobados

Fuente: Corporación Financiera Nacional Banca Pública Elaborado: Los autores 


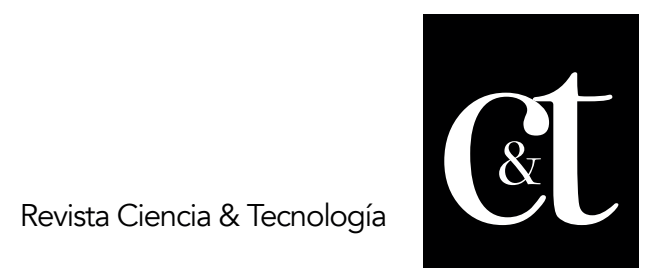

No. 28, 31 de octubre de 2020

ISSN impreso: 1390 - 6321

ISSN online: 2661 - 6734

Con los cálculos de cuadro anterior se determinó el error estándar con un resultado de 4,01878; un coeficiente $R^{2}$ igual a 0,40479 y una correlación igual a 0,63623; con esta información, se puede inferir:

El error estándar de 4,01878 determina que cualquier pronóstico podría sufrir una distorsión de +/- 4 operaciones anuales; que el coeficiente $R^{2}$ igual a 0,40479 implica un grado de certeza MEDIO en relación a las muestras lo que equivalente al 40,48\%; que la correlación igual a 0,63623 determina que los datos y sus pronósticos mantienen un $63,62 \%$ de correlación entre ellos.

La ecuación para pronósticos que se determina con los cálculos es:

$y=14,51+0,1235 x$

2. Regresión simple de los empleos vs montos de operaciones aprobadas

Previo a realizar el análisis de la regresión simple de los empleos vs. montos de operaciones aprobadas, se debe citar que dentro de la solicitud que cada persona natural y/o jurídica presenta para su requerimiento de crédito, este debe indicar cuántos empleados fijos y temporales posee al inicio de su solicitud de crédito y proyectar cual va a ser el impacto social del mismo, es decir, cuanto empleo generará, el mismo que es corroborado por el analista de crédito de la CFN e incluido en su informe de crédito, cuyos datos son tabulados por el área de planeación de la Institución.

Analizando los datos de los empleos generados de las operaciones aprobadas, según se muestran en la Tabla 2 de los datos de los empleos vs montos aprobados ambas al mismo tiempo para medir su correlación y certeza en el eje de las $X$ los montos en cientos de miles de US\$ (variable independiente) y en el eje de las Y los empleos generados por las operaciones aprobadas (variable dependiente).

Tabla 2. Datos de los empleos vs. montos aprobados

\begin{tabular}{|l|c|c|}
\hline Periodo & $\begin{array}{c}\text { Empleos (y) } \\
\text { (unidades) }\end{array}$ & $\begin{array}{c}\text { Montos (x) } \\
\text { (cientos de miles US\$) }\end{array}$ \\
\hline Año 2012 & 46 & 23,7 \\
\hline Año 2013 & 81 & 47,4 \\
\hline Año 2014 & 180 & 15,2 \\
\hline Año 2015 & 143 & 65,3 \\
\hline Año 2016 & 166 & 78,8 \\
\hline Intercepto & 96,08996 \\
Pendiente & 0,58849 \\
\hline
\end{tabular}

Fuente: Corporación Financiera Nacional Banca Pública

Elaborado: Los autores

Con los presentes datos, de igual manera, se podrán calcular los debidos pronósticos lineales en función del número de operaciones aprobadas por cada año; asimismo, el error explicado cuyas sumas se comprueban en cero en cuanto a su valor absoluto y el cuadrado de este. Estas cifras permiten apreciar el análisis de error de los datos en lo concerniente a los empleos y en lo que se vincula a montos aprobados. 
Según el Gráfico 2 sobre dispersión de empleos vs montos aprobados se muestra que estos estudios se basan en la creencia de que es posible identificar $\mathrm{y} / \mathrm{o}$ cuantificar alguna relación funcional entre dos o más variables, en la gráfica de dispersión se refleja una recta con pendiente positiva lo cual quiere decir, que su tendencia es creciente en el tiempo con un coeficiente de determinación $\mathrm{R}^{2}$ BAJO de 0,0759 .

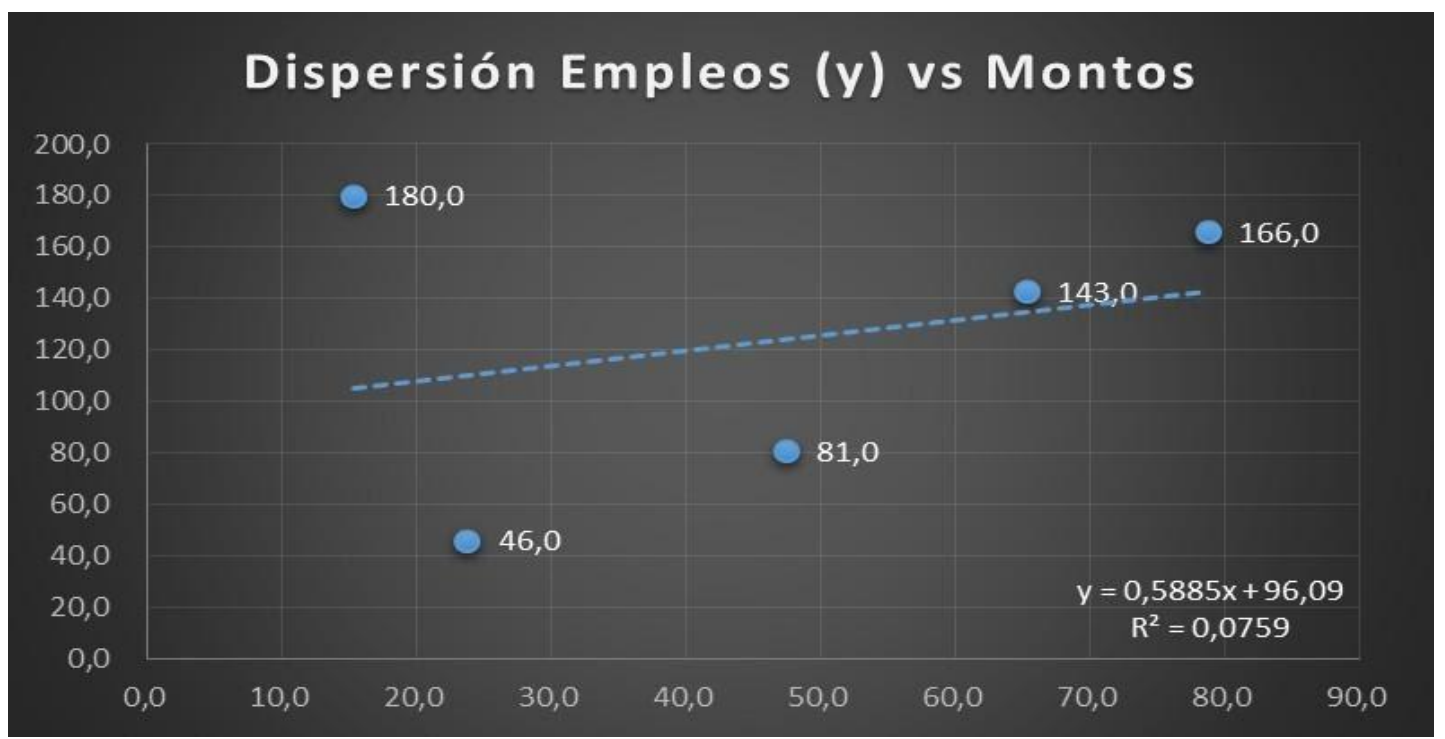

Gráfico 2. Dispersión de empleos vs montos aprobados

Fuente: Corporación Financiera Nacional Banca Pública

Elaborado: Los autores

Con los cálculos de cuadro anterior se determinó el error estándar con un resultado de 55,20465; un coeficiente $R^{2}$ igual a 0,07585 y una correlación igual a 0,27542; con esta información, se puede inferir que el error estándar de 55,20465 determina que cualquier pronóstico podría sufrir una distorsión en la cantidad +/- 55 empleos en función de los montos de crédito aprobadas hacia arriba o hacia abajo. Además, que el coeficiente $R^{2}$ igual a 0,07585 equivalente al $7,59 \%$ lo que implica un grado BAJO de certeza de las muestras y la información analizada; que la correlación igual a 0,27542 determina que los datos y sus pronósticos mantienen un $27,54 \%$ de correlación entre ellos, considerada BAJA.

La ecuación para pronósticos que se determina con los cálculos es:

$y=96,09+0,5885 x$

Según estos resultados, no es concluyente que, a mayores montos aprobados necesariamente se generen mayores empleos esperados (los empleos no agregan valor a la ecuación).

3. Regresión simple de los impuestos vs montos de las operaciones aprobadas Analizando los datos de impuestos generados de operaciones aprobadas, según se muestran en la tabla 3, al mismo tiempo para medir su correlación y certeza en el eje de las X los montos en cientos de miles de US\$ (variable independiente) y en el 
eje de las $\mathrm{Y}$ los impuestos generados por las operaciones aprobadas (variable dependiente).

Tabla 3. Datos de los impuestos vs. Montos aprobados

\begin{tabular}{|c|c|c|}
\hline Periodo & $\begin{array}{c}\text { Impuestos (y) } \\
\text { (miles US\$) }\end{array}$ & $\begin{array}{c}\text { Montos (x) } \\
\text { (cientos de miles US\$) }\end{array}$ \\
\hline Año 2012 & 0 & 23,7 \\
\hline Año 2013 & 59,5 & 47,4 \\
\hline Año 2014 & 0 & 15,2 \\
\hline Año 2015 & 215,0 & 65,3 \\
\hline Año 2016 & 142,5 & 78,8 \\
\hline $\begin{array}{l}\text { Intercepto } \\
\text { Pendiente }\end{array}$ & $-57,91097$ & \\
\hline
\end{tabular}

Fuente: Corporación Financiera Nacional Banca Pública

Elaborado: Los autores

El Gráfico 3 de dispersión de impuestos vs montos aprobados, muestra una relación funcional entre las dos variables, en la gráfica de dispersión se refleja una recta con pendiente positiva lo cual quiere decir, que su tendencia es creciente, con un coeficiente de determinación $\mathrm{R}^{2}$ ALTO de 0,7703, es decir los montos aprobados de crédito explican en un $77 \%$ las variaciones observadas en los impuestos generados.

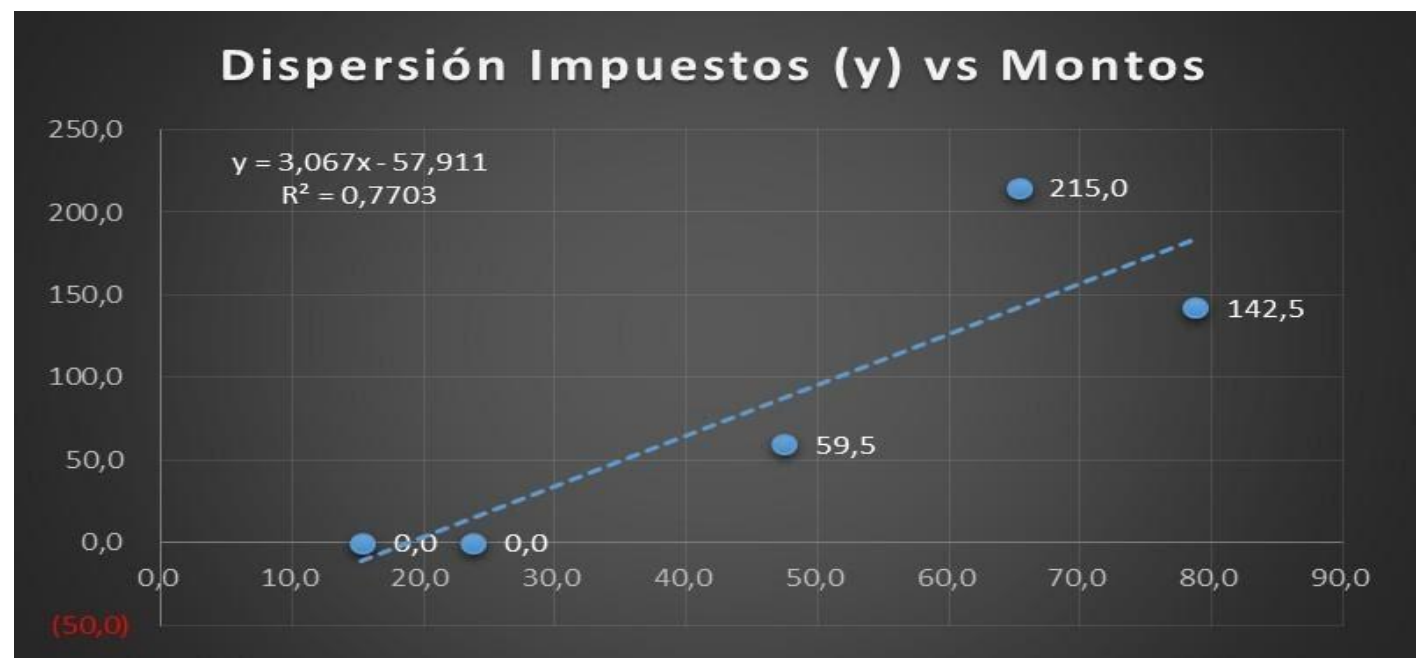

Gráfico 3. Dispersión de impuestos generados vs montos de las operaciones aprobadas

Fuente: Corporación Financiera Nacional Banca Pública Elaborado: Los autores

Con los cálculos de cuadro anterior se determinó el error estándar con un resultado de 45,01639; un coeficiente $\mathrm{R}^{2}$ igual a 0,77027 y una correlación igual a 0,87765; con esta información, se puede inferir:

Que el error estándar de 45,01639 determina que cualquier pronóstico podría sufrir una distorsión en la cantidad +/- US\$ 45.016 impuestos en función de los montos de crédito aprobadas hacia arriba o hacia abajo; que el coeficiente $R^{2}$ igual a 0,77027 equivalente al $77,03 \%$ lo que implica un grado ALTO de certeza de las muestras y la 


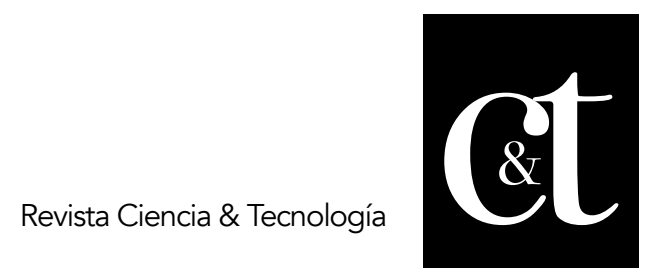

No. 28, 31 de octubre de 2020

ISSN impreso: 1390 - 6321

ISSN online: 2661 - 6734

información analizada; y que la correlación igual a 0,87765 determina que los datos y sus pronósticos mantienen un $87,77 \%$ de correlación entre ellos, considerada ALTA.

La ecuación para pronósticos determinada con los cálculos es:

$y=-57,911+3,067 x$

Todo lo anterior confirma que a mayor monto aprobado mayor impuesto esperado.

Pronóstico de las ecuaciones

A continuación, en base a las ecuaciones estimadas de regresión simple de número de operaciones vs montos aprobados, de los empleos vs montos de operaciones aprobadas, y de los impuestos vs montos de las operaciones aprobadas, se procede a realizar los respectivos pronósticos de las variables dependientes.

1. Ecuación de regresión simple montos y números de operaciones aprobadas Con la definición de la ecuación $\mathrm{y}=14,51234+0,12346 *(\mathrm{x})$ se calcula el pronóstico de regresión simple de los números de operaciones aprobadas (y) tomando como ( $\mathrm{x}$ ) los montos, obsérvese la Tabla 4 sobre Pronóstico del número de operaciones aprobadas:

\section{Tabla 4. Pronóstico del número de operaciones aprobadas}

Ecuación: $\quad y=14,51234+0,12346 *(x)$
\begin{tabular}{|c|c|c|c|}
\hline \multirow{2}{*}{ Período } & \multirow{2}{*}{$\begin{array}{c}\text { Operaciones } \\
\text { (unidades) }\end{array}$} & \multicolumn{2}{|c|}{ Montos } \\
\cline { 3 - 4 } & 26 & 90,0 & $9.000 .000,0$ \\
\hline 2017 & 29 & 120,0 & $12.000 .000,0$ \\
2018 & 29 & 150,0 & $15.000 .000,0$ \\
2019 & 33 & 200,0 & $20.000 .000,0$ \\
\cline { 2 - 4 } 2020 & 39 & 250,0 & $25.000 .000,0$ \\
\cline { 2 - 4 } 2021 & 45 & & \\
\cline { 2 - 4 }
\end{tabular}

Elaborado: Los autores

Este cuadro del pronóstico del número de operaciones aprobadas en base a la ecuación $\mathrm{y}=14,51234+0,12346 *(\mathrm{x})$ proyectando $\mathrm{X}$ en formato en cientos de miles 90,0 que es el equivalente a 9 millones de dólares, pronostica 26 operaciones para el año 2017, y así sucesivamente.

A continuación, se ilustra en el Gráfico 4 el pronóstico del número de operaciones aprobadas. 


\section{\# OPERACIONES VS MONTOS (X)}

50
45
40
35
30
25
20
15
10
5
0

\section{Gráfico 4. Pronóstico del número de operaciones aprobadas}

Elaborado: Los autores

2. Ecuación de regresión simple de los empleos vs montos de operaciones aprobadas

Con la definición de la ecuación $y=96,08996+0,58849 *(x)$ se calcula el pronóstico de regresión simple de los empleos (y) tomando como (x) los montos, obsérvese la Tabla 5 sobre el pronóstico de los empleos:

\section{Tabla 5. Pronóstico de los empleos}

\begin{tabular}{|c|c|c|c|}
\hline \multirow{2}{*}{ Período } & \multirow{2}{*}{$\begin{array}{l}\text { Empleos } \\
\text { (unidades) }\end{array}$} & \multicolumn{2}{|c|}{ Montos } \\
\hline & & (cientos de miles US\$) & (US\$) \\
\hline 2017 & 149 & 90,0 & $9.000 .000,0$ \\
\hline 2018 & 167 & 120,0 & $12.000 .000,0$ \\
\hline 2019 & 184 & 150,0 & $15.000 .000,0$ \\
\hline 2020 & 214 & 200,0 & $20.000 .000,0$ \\
\hline 2021 & 243 & 250,0 & 25.000 .000 .0 \\
\hline
\end{tabular}

Elaborado: Los autores

Este cuadro del Pronóstico del número de empleos basado en la ecuación y = $96,08996+0,58849 *(x)$ proyectando $X$ en formato en cientos de miles 90,0 que es el equivalente a 9 millones de dólares, pronostica 149 empleos para el año 2017, así sucesivamente.

A continuación, el Gráfico 5 muestra el pronóstico de los empleos. 


\section{EMPLEOS VS MONTOS (X)}

300

250

200

150

100

50

0

\section{Gráfico 5. Pronóstico de los empleos}

Elaborado: Los autores

3. Ecuación de regresión simple de los impuestos vs montos de las operaciones aprobadas

Con la definición de la ecuación $y=-57,91097+3,06704 *(x)$ se calcula el pronóstico de regresión simple de los impuestos $(y)$ tomando como $(x)$ los montos, obsérvese la Tabla 6 de los pronósticos de los empleos:

\section{Tabla 6. Pronóstico de los impuestos}

\begin{tabular}{|l|c|c|c|}
\hline \multirow{3}{*}{ Ecuación: } & $y=-57,91097+3,06704 *(x)$ \\
\hline 2017 & \multirow{2}{*}{$\begin{array}{c}|c| \\
\text { Impuestos }\end{array}$} & \multicolumn{2}{|c|}{ Montos } \\
\cline { 3 - 4 } 2018 & 218,1 & 90,0 & $9.000 .000,0$ \\
2019 & 310,1 & 120,0 & $12.000 .000,0$ \\
\cline { 3 - 4 } 2020 & 402,1 & 150,0 & $15.000 .000,0$ \\
\cline { 2 - 4 } 2021 & 555,5 & 200,0 & $20.000 .000,0$ \\
\cline { 2 - 4 } & 708,8 & 250,0 & $25.000 .000,0$ \\
\hline
\end{tabular}

Elaborado: Los autores

Este cuadro del Pronóstico de los impuestos tomado de la ecuación y $=-57,91097$ $+3,06704 *(x)$ proyectando $X$ en formato en cientos de miles 90,0 que es el equivalente a 9 millones de dólares, pronostica 218,1 mil dólares en impuestos para el año 2017, y así sucesivamente.

A continuación, se grafica el pronóstico de los impuestos. 


\section{IMPUESTOS VS MONTOS (X)}

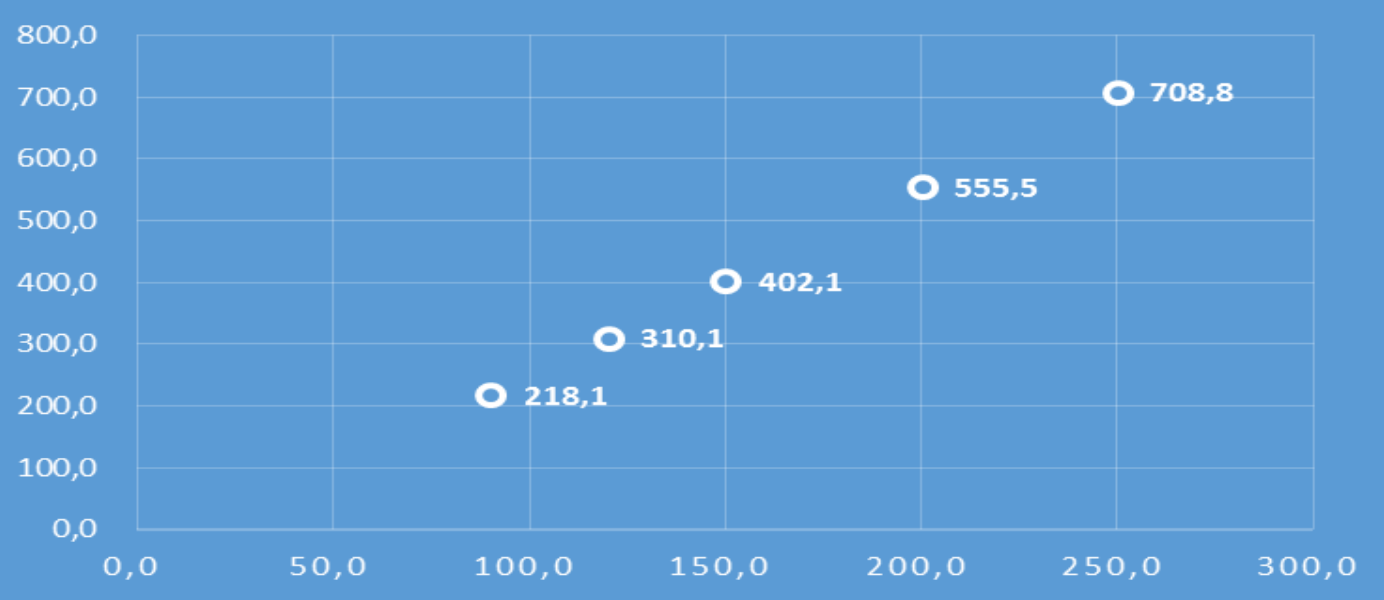

\section{Gráfico 6. Pronóstico de los impuestos}

Elaborado: Los autores

Lo revisado previamente indica que, de hecho, es posible realizar el pronóstico de los impuestos a base de los datos obtenidos.

\section{Discusión}

Con la definición de la ecuación y $=14,51234+0,12346 *(x)$ se calculó el pronóstico de regresión simple de los números de operaciones aprobadas ( $y$ ) tomando como $(x)$ los montos.

El error estándar de 4,01878 determina que cualquier pronóstico podría sufrir una distorsión de +/- 4 operaciones anuales; Que el coeficiente $R^{2}$ igual a 0,40479 implica un grado de certeza de nivel MEDIO en relación a las muestras lo que equivalente al 40,48\%; Que la correlación igual a 0,63623 determina que los datos y sus pronósticos mantienen un $63,62 \%$ de correlación entre ellos.

Con la ecuación y $=96,08996+0,58849 *(x)$ se calculó el pronóstico de regresión simple de los empleos (y) tomando como ( $\mathrm{x}$ ) los montos; que el error estándar de 55,20465 determina que cualquier pronóstico podría sufrir una distorsión en la cantidad +/- 55 empleos en función de los montos de crédito aprobadas hacia arriba o hacia abajo; el coeficiente $\mathrm{R}^{2}$ igual a 0,07585 equivalente al $7,59 \%$ lo que implica un grado BAJO de certeza de las muestras y la información analizada.

La correlación igual a 0,27542 determina que los datos y sus pronósticos mantienen un $27,54 \%$ de correlación entre ellos, considerada BAJA; lo que quiere decir, que no se explica que a mayores montos aprobados no necesariamente se generarían mayores empleos, en otras palabras, los empleos no agregan valor a la ecuación.

Con la definición de la ecuación $\mathrm{y}=-57,91097+3,06704 *(\mathrm{x})$ se calculó el pronóstico de regresión simple de los impuestos $(y)$ tomando como $(x)$ los montos; el error estándar de 45,01639 determina que cualquier pronóstico podría sufrir una distorsión en la cantidad +/- US\$ 45.016 de impuestos en función de los montos de crédito aprobadas hacia arriba o hacia abajo; el coeficiente $R^{2}$ igual a 0,77027 


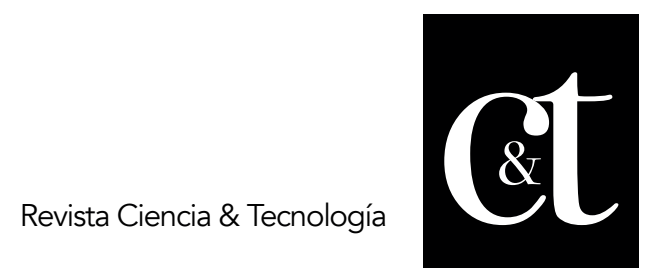

No. 28, 31 de octubre de 2020

ISSN impreso: 1390 - 6321

ISSN online: 2661 - 6734

equivalente al $77,03 \%$ lo que implica un grado ALTO de certeza de las muestras y la información analizada; la correlación igual a 0,87765 determina que los datos y sus pronósticos mantienen un $87,77 \%$ de correlación entre ellos, considerada ALTA; todo lo anterior confirma que a mayores montos aprobados mayor impuestos esperados.

\section{Conclusiones}

Para este trabajo se tomaron las variables $\mathrm{X}_{1}$ : Fomentar la asesoría financiera $\mathrm{y}$ tributaria como independiente y las variables $Y_{1}$ : Créditos directos para personas naturales, $Y_{2}$ : Nuevas plazas de trabajos y $Y_{3}$ : Tributos fiscales como dependientes, y se realizaron regresiones lineales simples por separado.

Con los resultados de las regresiones estimadas se pudo inferir que mientras mayor sea el número de empleados que se tenga y mayores tributos pague el cliente, mayor línea de crédito podrá acceder. En este caso, lo que aplica es la regresión lineal simple, medida cada variable por separado, porque cada variable analizada no se correlaciona para el objetivo de comprobar la hipótesis, es decir, el nivel operaciones aprobadas, no tiene correlación ni agrega valor con el número de empleos ni con los impuestos en conjunto.

No hay que descartar que, en este tipo de análisis, podría aplicarse la regresión lineal múltiple, para lo cual es necesario identificar adecuadamente las variables dependientes o explicativas y la variable dependiente. Se debe contrastar con los resultados y cálculos de coeficiente $R^{2}$ y correlación o determinación. Cuando se presente un caso de investigación con más de dos variables se deben contrastar ambos modelos por separado y analizar los resultados con la finalidad de determinar qué modelo aplica con mayor confiabilidad en su pronóstico.

\section{Referencias bibliográficas}

Asamblea Nacional del Ecuador. (2014). Código orgánico monetario y financiero. Quito: Asamblea Nacional del Ecuador.

Berenson, M. L., Levine, M., \& Krehbiel, C. (2006). Estadística para administración. Ciudad de México: Instituto Tecnológico y Estudios Superiores de Monterrey.

Emery, D. R., Finnerty, D., \& Stowe, D. (2000). Fundamentos de administración financiera. México DF: Pearson Educación.

Estrada, J. (2012). Análisis de los obstáculos financieros de las pymes para la obtención de crédito y las variables que lo facilitan. Valencia: Universidad Politécnica Vaca de Valencia.

Ismail, S. (2013). Determinants of Personal Loans Borrowing: An Empirical Study. 2013 IEEE Symposium on Business, Engineering and Industrial Applications. Conference Paper.

Kon, I., \& Storey, D. (2003). A theory of discouraged borrowers. Small Business Economics. 21, 37-49.

Navarro Castano, D. (2003). Administración Financiera. Manizales: Universidad Nacional de Colombia.

Peñarreta, M., García, D. \& Armas, R. (2019). Educación financiera y factores determinantes: Evidencias desde Ecuador. Revista Espacios 40(7). 


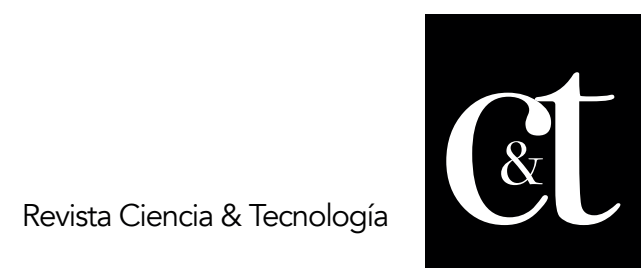

No. 28, 31 de octubre de 2020

ISSN impreso: 1390 - 6321

ISSN online: 2661 - 6734

Samaniego, R., Trujillo, A. \& Martin, J. (2006). Un Análisis de los modelos contables y de mercado en la evaluación del riesgo de crédito: aplicación al mercado bursátil español. Madrid: Revista Europea de Dirección y Economía de la Empresa, Vol. 16 No. 2: 93-110.

Servicio de Rentas Internas SRI (2016). Rendición de Cuentas. Quito: SRI.

Trujillo Ponce, A. (2002). Gestión del riesgo de crédito en préstamos comerciales. Madrid: Instituto Superior de Técnicas y Prácticas Bancarias. 